\title{
Hidatidosis humana: antecedentes generales y situación epidemiológica en Chile, 2001-2009
}

\author{
Paulina Martínez G.
}

\section{Human hydatidosis disease: general background and epidemiological situation in Chile, 2001-2009}

Objective: To characterize the epidemiology of human hydatidosis in Chile. Material: Data from the Mandatory Disease Notification System (2001-2009), hospital discharges (2001-2008), Deaths (2001-2008) and Years of Potential Life Lost (2001-2008) were analyzed. Results: The average incidence, according to the mandatory notification data, was 1.9 cases per 100,000 inhabitants (men 51.4\%). Median age was 38 years. Hospital discharge rate for the period was 6.3 discharges per 100,000 inhabitants. The main diagnosis corresponded to B67.9 (Echinococcosis, other and unspecified). The average mortality rate was 0.2 deaths per 100,000 inhabitants. The level of education of fatalities was mainly basic. There was a loss of 3.349 years of life due to the premature death of 235 people. Conclusions: The incidence rates of cases reported, hospital discharges and mortality tend to decrease. There is a noteworthy discrepancy between reported cases and hospital discharges, which suggests underreporting. Improvement of the notification system and additional prevalence studies are required.

Key words: Hydatidosis; human hydatidosis; cases reported; hospital discharges; deaths; PPYLL.

Palabras clave: Hidatidosis; hidatidosis humana; casos notificados; egresos hospitalarios; defunciones; AVPP.

\section{Introducción}

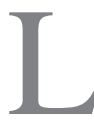

a hidatidosis es una zoonosis causada por formas larvarias del género Echinococcus. Existen diferentes especies de Echinococcus, pero sólo cuatro -E. granulosus, E. multilocularis, E. oligarthrus y E. vogeli-son reconocidas como taxonómicamente relevantes y sólo las primeras dos son patogénicas para el humano ${ }^{1}$.

La hidatidosis se encuentra ampliamente distribuida a nivel mundial, alcanzando alta incidencia en países como Argentina, Uruguay, Chile, Australia, Nueva Zelandia, y en Europa, fundamentalmente en Grecia, Italia, Portugal y España ${ }^{2,3}$.

Por ser una enfermedad que genera alto impacto socio-económico por días no trabajados, cirugía y recuperación, exámenes, medicamentos, consultas médicas y prestaciones anexas entre otros, el Ministerio de Salud de Chile (MINSAL) incorporó esta enfermedad al sistema de notificación obligatoria.

El presente trabajo presenta las características generales de la enfermedad en Chile y los principales indicadores de esta enfermedad, según el Sistema de Notificación Obligatoria (ENO), Egresos Hospitalarios, Defunciones y Años de Vida Potencialmente Perdidos (AVPP).

\section{Características generales}

La equinococosis humana es una enfermedad zoonótica causada por formas larvarias de varios géneros del parásito cestodo Echinococcus. El ciclo de vida del parasito comprende dos hospederos: definitivo o carnívoro, donde los parásitos adultos se desarrollan en el intestino; hospedero intermediario-herbívoro u omnívoro- que presenta las formas larvarias en los tejidos. El hombre está dentro de este último grupo, como hospedero accidental ${ }^{4}$.

Las especies E. granulosus y E. multilocularis, son patogénicas para humanos. Para distinguir la enfermedad causada por estas dos diferentes especies, la Organización Mundial de la Salud (OMS) propuso la designación de “quiste equinococócico" para la enfermedad causada por E. granulosus y equinococosis alveolar para la enfermedad causada por E. multilocularis ${ }^{1}$.

El quiste equinococócico es el más frecuente y está producido por la fase larval del E. granulosus. Durante el curso natural de la enfermedad, la fase del quiste hidatídico es variable. Algunos quistes pueden crecer a un promedio de 1-30 mm por año y pueden pasar muchos años sin transformación. Puede producirse una rotura natural y desaparecer completamente ${ }^{1}$.

El período de incubación es variable y no ha sido definido. La infección puede ser sintomática dependiendo de la ubicación del quiste y sus efectos sobre los tejidos adyacentes induciendo síntomas clínicos. Una de las complicaciones más frecuentes es la rotura del quiste -espontánea, traumática o durante procedimientos de intervención- que produce reacción anafiláctica y formación de quistes secundarios. Usualmente los quistes

\author{
Universidad Diego Portales, \\ Santiago. Chile. \\ Facultad de Medicina. \\ Escuela de Tecnología Médica. \\ Financiamiento: el estudio se realizó \\ sin aporte económico o becas. \\ La autora declara no tener \\ conflictos de interés con este \\ artículo. \\ Recibido: 28 de febrero de 2011 \\ Aceptado: 15 de agosto de 2011 \\ Correspondencia a: \\ Paulina Martínez Gallegos \\ paulinamartinezg@yahoo.com
}


no inducen síntomas clínicos hasta alcanzar un tamaño determinado ${ }^{5}$.

La mayoría de los pacientes (sobre 80\%) tienen un solo órgano afectado y con un quiste único. Alrededor de dos tercios de los casos se ubican en el hígado y cerca de $20 \%$ en los pulmones ${ }^{1,6}$.

El diagnóstico en el ser humano se realiza básicamente por antecedentes clínicos y epidemiológicos, con apoyo de imágenes radiológicas. La observación de los quistes por laparoscopía, laparotomía u otros procedimientos visuales, contribuye a perfeccionar la tesis diagnostica. Las pruebas serológicas proporcionan un diagnóstico específico pero, para que se produzcan debe haber una reacción antígeno/anticuerpo, es decir, fisura o rotura de la capa germinativa del quiste. Como ninguna técnica por si sola otorga un diagnostico certero, es necesario la asociación entre ellas².

Últimamente, se recurrió al examen bioquímico y uso de marcadores enzimáticos, seguidos de técnicas de biología y genética molecular que permitieron avanzar en la discriminación de las especies reconocidas de Echinococcus, teniendo implicaciones de gran importancia para el diseño de los programas de control ${ }^{2}$.

Tradicionalmente ha sido considerada una enfermedad con resolución quirúrgica, donde se elimina el (los) quiste(s), corrección de efectos en el órgano afectado y tratamiento de posibles complicaciones ${ }^{3}$. En la actualidad se dispone de un tratamiento ecoasistido para el quiste hepático basado en la punción del quiste, aspiración de parte del contenido e introducción de una sustancia escolicida. Esta solución luego es totalmente aspirada. Esta técnica es conocida como PAIR (Puncture, Aspiration, Injection (of the scolicide) and Reaspiration) ${ }^{7}$.

\section{Situación epidemiológica}

Enfermedad de importancia en salud pública, donde actúan diversas causas que modifican la dinámica de transmisión, como la gran adaptación a diferentes perfiles geográficos con diversos nichos ecológicos y culturales ${ }^{8}$.

La equinococosis humana es una enfermedad con altos costos socioeconómicos, que incluye pérdidas por días de ausencia laboral, hospitalización, cirugías, tratamientos médicos, exámenes y consultas médicas especializadas, entre otras ${ }^{2}$.

Considerada una enfermedad crónica, es más fácil de adquirir en los primeros años de vida, dado los hábitos de los menores de edad, lo cual los expone al riesgo de contraer huevos desde las heces de los hospederos definitivos ${ }^{9}$.

De distribución mundial, con países endémicos como Perú, Brasil, Uruguay, Argentina y Chile $^{10}$, asociada a zonas rurales dedicadas al pastoreo, siendo el hospedero intermediario animal de mayor importancia epidemio- lógica el ovino; sin embargo, también pueden adquirir importancia relativa otras especies animales, como cerdos, caprinos, camélidos sudamericanos. Los bovinos y equinos generalmente no representan una fuente importante de infección para los perros ${ }^{11}$.

En Chile, es una enfermedad de notificación obligatoria (ENO) de acuerdo al Decreto Supremo No 712, año 2000. El objetivo del sistema de vigilancia es caracterizar epidemiológicamente los casos, forma de presentación y fuentes de infección, de manera de interrumpir la transmisión y desarrollar medidas de prevención y control en humanos ${ }^{12}$.

De acuerdo a la Norma Técnica $N^{\circ} 55$, sobre Vigilancia de Enfermedades Transmisibles, el caso confirmado corresponde al caso sospechoso confirmado por anatomía patológica o visualización directa por microscopia de proto-escólices del cestodo ${ }^{13}$.

El sistema ENO establece la obligación de notificar diariamente la sospecha de hidatidosis a la Autoridad Sanitaria Regional.

La enfermedad afecta a todas las edades pero, particularmente edades productivas, repercutiendo fuertemente sobre el individuo afectado, su entorno familiar y sobre la economía nacional ${ }^{14}$.

Las defunciones son muertes evitables, dado que es una enfermedad que tiene medidas de control, normas de diagnóstico y tratamiento. El impacto económico que genera la equinococosis quística en las personas, se mide a través de los costos que generan la morbilidad, mortalidad y AVPP. Las muertes por hidatidosis entre la gente joven producen mayor pérdida de años de vida que las muertes que se producen entre las personas adultas mayores y consecuentemente mayor pérdida en años de producción para la sociedad.

\section{Métodos}

Estudio descriptivo. Se emplearon los datos secundarios provenientes del Sistema ENO del MINSAL, entre los años 2001 y 2009 (siendo el 2009 el último con datos validados). Se distinguieron según la Clasificación Internacional de Enfermedades (CIE10) los códigos B67.0 (Infección del hígado debida a E. granulosus); B67.1 (Infección del pulmón debida a E. granulosus); B67.2 (Infección de hueso debida a E. granulosus); B67.3 (Infección de otro órgano y de sitios múltiples por $E$. granulosus); B67.4 (Infección debida a E. granulosus, sin otra especificación); B67.5 (Infección del hígado debida a E. multilocularis); B67.6 (Infección de otros órganos y de sitios múltiples por E. multilocularis); B67.7 (Infección debida a E. multilocularis, sin otra especificación); B67.8 (Equinococosis del hígado, no especificada); B67.9 (Equinococosis, otra y la no especificada) ${ }^{15}$. 
Se utilizaron los Egresos Hospitalarios, 2001-2008 (siendo este último año preliminar) ${ }^{16}$.

También se empleó el Registro de Mortalidad Nacional 2001-2008.

Se estimaron los AVPP 2001-2008, por años, sexo y grupos de edad, considerando la esperanza de vida del Instituto Nacional de Estadísticas (INE) ${ }^{17}$.

En los casos ENO, egresos hospitalarios y defunciones, se calcularon las tasas por año y región, utilizando la población INE ${ }^{18}$.

Mediante estadísticos descriptivos se describieron las características de las personas como sexo, edad, diagnóstico, condición de egreso, nivel de educación, intervención quirúrgica.

Se evalúo la variación temporal y geográfica de las tasas de incidencia ENO, egresos hospitalarios y defunciones.

\section{Resultados}

\section{Vigilancia ENO}

Los casos han variado desde 297 en 2001 (52,2\% mujeres) a 267 en 2009 (52,4\% hombres), con un promedio anual de 311 casos.

La tasa de notificación se ha desplazado desde 1,9 casos por 100 mil habs., en 2001 a 1,6 casos por 100 mil habs, en 2009 (Figura 1), evidenciando una tendencia al decrecimiento.

Las Tablas 1 y 2 muestran las principales características de las personas afectadas y su distribución por sexo y grupos de edad.

La edad mediana de notificación fue 38 años; hombres 39 años (0-94 años); mujeres 37 años (0-96 años).

Según diagnóstico CIE10, 28,2\% de los casos notificados son clasificados como B67.0.

Respecto a la distribución geográfica, las mayores tasas de incidencia se registran en Aysén (XI Región) con 327 casos por 100 mil habs (Figura 2).

\section{Egresos hospitalarios}

Los egresos han variado de 1.150 en 2001 (53,2\% hombres) a 498 en 2008 (52,4\% hombres), con un promedio anual cercano a 1.009 egresos. El mayor número de egresos se registró en $2003(\mathrm{~N}=1.224)$.

La tasa de egresos hospitalarios se ha desplazado desde 7,4 a 3 egresos por 100 mil habs, con tendencia al decrecimiento (Figura 1).

Las Tablas 1 y 2 muestran las principales características de las personas afectadas y su distribución por sexo y grupos de edad.

La edad mediana de notificación fue 40 años (0-105 años); hombres 40 años (0-94 años) y; mujeres 30 años (0-105 años).

Según el tiempo de estadía, se registra una mediana

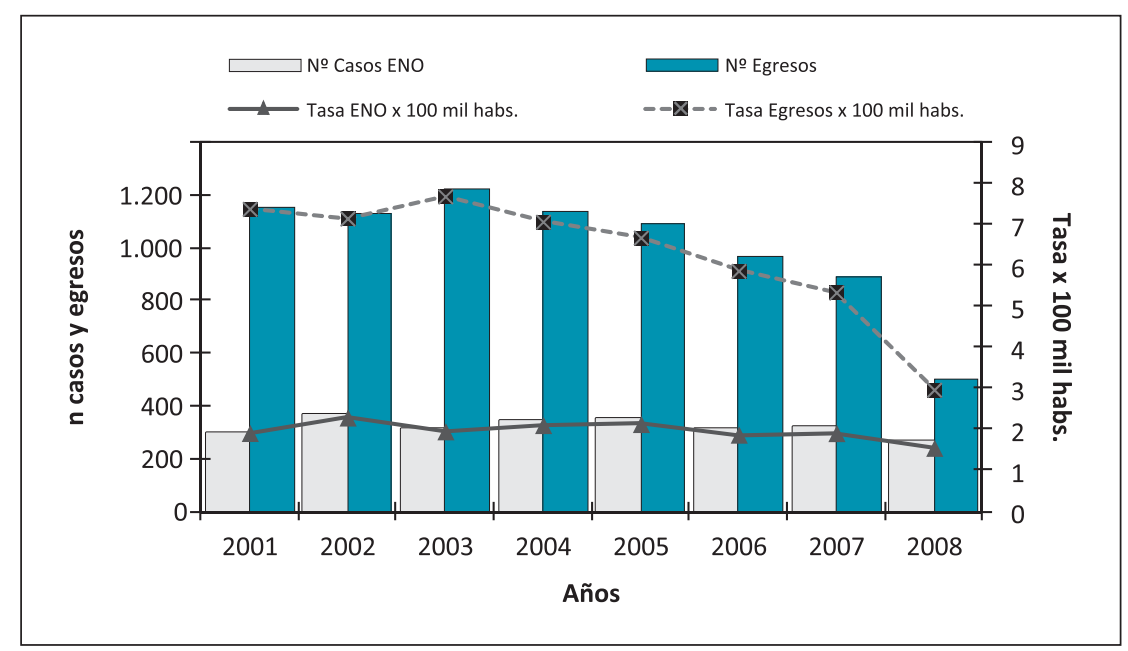

Figura 1. Casos notificados (ENO) y Egresos hospitalarios de hidatidosis humana. Chile, 2001-2008.

de 9 días (1-189); hombres 9 días (1-153); mujeres 8 días (1-189).

Según diagnóstico CIE10, 46\% son clasificados como B67.9.

Según las tasas de incidencia regional, la mayor es en Aysén con 368,6 egresos por 100 mil habs (Figura 3) .

\section{Mortalidad}

Las muertes han variado de 31 en 2001 (61,3\% hombres) a 22 en 2008 (63,6\% hombres), con un promedio anual cercano a 29,4 defunciones. La tasa de mortalidad evidencia una tendencia al decrecimiento (Figura 4).

La edad mediana de muerte fue 68 años (3-97 años); hombres 68 años (12-94 años); mujeres 70 años (3-97 años).

Según diagnóstico CIE10, 50,6\% son clasificados como B67.9.

De acuerdo a las tasas de mortalidad regional, la mayor es en Araucanía con 6,3 defunciones por 100 mil habs. (Figura 4).

Conforme el estado civil al momento de fallecer, 47,2\% se encuentra casado y $40,4 \%$ soltero.

De conformidad al nivel de instrucción de los fallecidos, 53,2\% registra un nivel escolar básico, mientras $16,9 \%$ no presenta escolaridad.

La Tabla 3 presenta el número de defunciones y tasas de mortalidad, del Registro Nacional de Mortalidad y según condición en los egresos hospitalarios. En ambas, se observa una disminución.

\section{Años de vida potencialmente perdidos}

Se produjo la pérdida de 3.854,99 años de vida por el fallecimiento prematuro de 235 personas. Este es el impacto que produjo la zoonosis sobre la mortalidad en AVPP y consecuentemente en años de producción para el país.

Los AVPP han disminuido 60,2\% entre el año 2001 
Tabla 1. Casos notificados (ENO) y egresos hospitalarios de hidatidosis humana según sexo. Chile, 2001-2009*

\begin{tabular}{|c|c|c|c|c|c|c|}
\hline Sexo & $\begin{array}{c}\text { n casos notificados (ENO) } \\
2001-2009\end{array}$ & $\%$ & Tasa $x 100$ mil habs. & $\begin{array}{c}\text { n egresos hospitalarios } \\
2001-2008\end{array}$ & $\%$ & Tasa $x 100$ mil habs. \\
\hline Hombre & 1.438 & 51,4 & 17,9 & 4.233 & 52,4 & 52,9 \\
\hline Mujer & 1.360 & 48,6 & 16,6 & 3.842 & 47,6 & 47,0 \\
\hline Total & 2.800 & 100,0 & 17,2 & 8.075 & 100,0 & 49,9 \\
\hline
\end{tabular}

\begin{tabular}{|c|c|c|c|c|c|c|}
\hline $\begin{array}{l}\text { Edad } \\
\text { (años) }\end{array}$ & $\begin{array}{c}\text { n casos notificados (ENO) } \\
2001-2009\end{array}$ & $\%$ & Tasa $x 100$ mil habs. & $\begin{array}{c}\text { n egresos hospitalarios } \\
2001-2008\end{array}$ & $\%$ & Tasa x 100 mil habs. \\
\hline $0-9$ & 299 & 10,7 & 11,7 & 640 & 7,9 & 24,7 \\
\hline 10-19 & 422 & 15,1 & 14,3 & 1.060 & 13,1 & 36,1 \\
\hline $20-29$ & 328 & 11,7 & 13,2 & 1.045 & 12,9 & 42,0 \\
\hline $30-39$ & 424 & 15,1 & 17,1 & 1.268 & 15,7 & 51,1 \\
\hline $40-49$ & 438 & 15,6 & 18,6 & 1.251 & 15,5 & 54,1 \\
\hline 50-59 & 362 & 12,9 & 23,2 & 1.187 & 14,7 & 77,3 \\
\hline $60-69$ & 287 & 10,3 & 28,4 & 881 & 10,9 & 88,6 \\
\hline 70-79 & 185 & 6,6 & 30,6 & 573 & 7,1 & 96,3 \\
\hline $80 y+$ & 55 & 2,0 & 21,8 & 170 & 2,1 & 68,6 \\
\hline Total & 2.800 & 100,0 & 17,2 & 8.075 & 100,0 & 49,9 \\
\hline
\end{tabular}

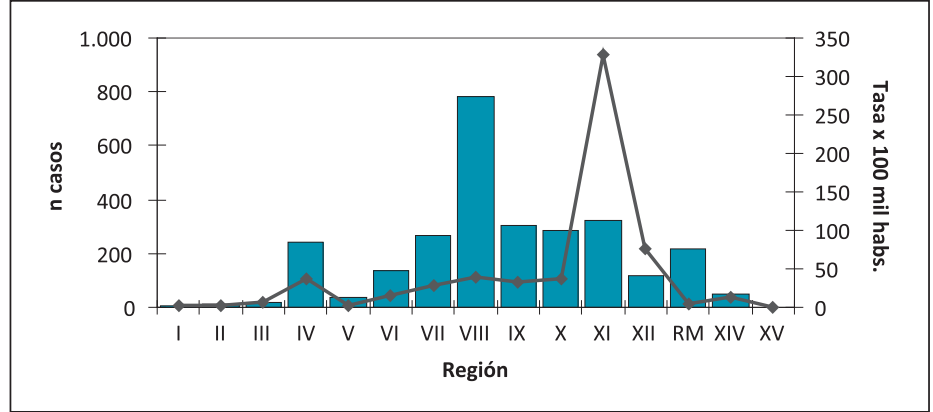

Figura 2. Variación geográfica de los casos y tasas de incidencia de hidatidosis (ENO). Chile, 2001-2009.

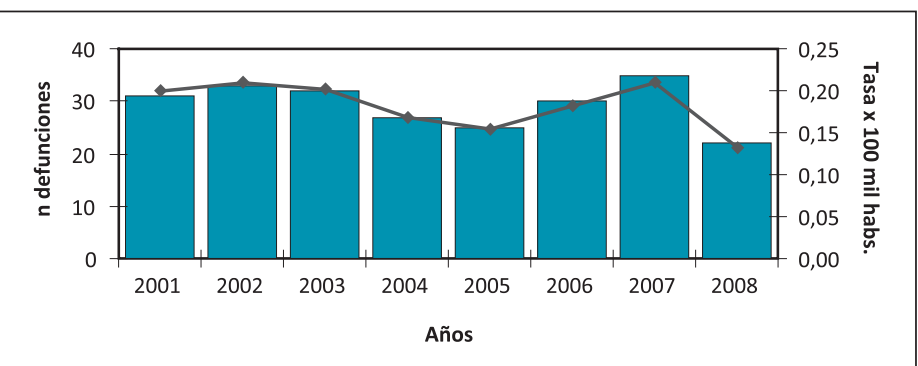

Figura 4. $N^{0}$ de defunciones y tasa de mortalidad por hidatidosis humana. Chile, 2001-2008.

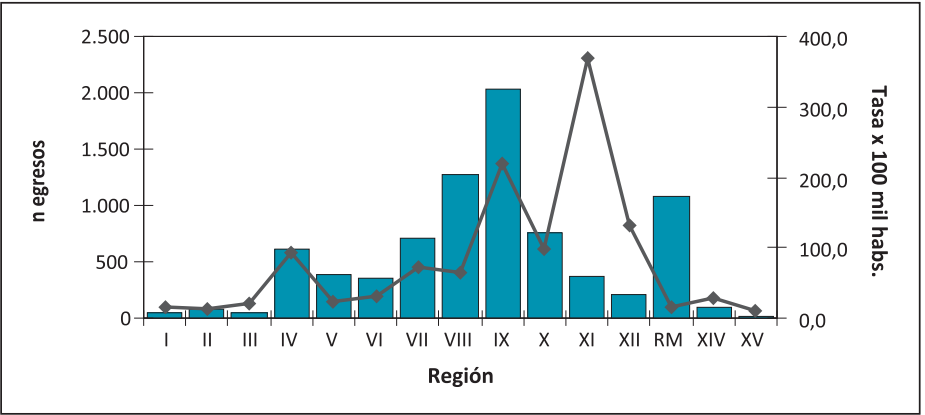

Figura 3. Variación geográfica del número y tasas de egresos hospitalarios de hidatidosis humana. Chile, 2001-2008.

$($ AVPP $=646,28)$ y $2008($ AVPP $=257,4)$. Los hombres $(1.932,82)$ perdieron más AVPP que las mujeres (1850,07).

A nivel país los grupos más afectados son: 50-59 años (19,7\%); 3039 años (19\%); y 20-29 años (15,1\%). En hombres: 30-39 años (27\%); 50-59 años (23,7\%); y 40-49 años (17,1\%). En mujeres: 20-29 años (21,3\%); 60-69 años (16,6\%); y 10-19 años (14,3\%).

Se observa que los $<20$ años favorecen a los hombres, ya que pierden menos AVPP en ese período de edad, respecto de las mujeres. Entre 20-59 años, corresponde a los hombres la mayor pérdida de AVPP. A partir de los 60 años, son las mujeres quienes pierden comparativamente más AVPP. 
Tabla 3. Defunciones y tasa de mortalidad de hidatidosis humana, según Registro Nacional y condición de egresos hospitalarios Chile, 2001-2008

\begin{tabular}{|c|c|c|c|c|c|}
\hline Años & $\begin{array}{l}n \text { defunciones } \\
\text { Registro Nacional }\end{array}$ & Tasa por 100.000 habs. & $\begin{array}{c}n \text { defunciones } \\
\text { egresos hospitalarios }\end{array}$ & Tasa por 100.000 habs. & $\begin{array}{c}\% \text { muertes en egresos } \\
\text { hospitalarios }\end{array}$ \\
\hline 2001 & 31 & 0,20 & 13 & 0,08 & 41,9 \\
\hline 2002 & 33 & 0,21 & 16 & 0,10 & 48,5 \\
\hline 2003 & 32 & 0,20 & 12 & 0,08 & 37,5 \\
\hline 2004 & 27 & 0,17 & 6 & 0,04 & 22,2 \\
\hline 2005 & 25 & 0,15 & 11 & 0,07 & 44,0 \\
\hline 2006 & 30 & 0,18 & 10 & 0,06 & 33,3 \\
\hline 2007 & 35 & 0,21 & 6 & 0,04 & 17,1 \\
\hline 2008 & 22 & 0,13 & 4 & 0,02 & 18,2 \\
\hline
\end{tabular}

\section{Discusión}

Las personas más afectadas por hidatidosis son hombres, concentrando 51,4 y 52,4\% según casos ENO y egresos hospitalarios, respectivamente.

Los afectados poseen mayoritariamente entre 30 y 59 años de edad. Este grupo de edad se encuentra en plena actividad productiva en términos laborales. Se ha de considerar que la población chilena esta más envejecida, lo cual significa que ha cambiado el índice de dependencia demográfica, por lo cual, al ser menor la proporción de personas en edades laborales, disminuye la oportunidad para incrementar la productividad nacional. Por lo tanto, esta enfermedad está asociada a pérdidas económicas importantes y no sólo a nivel humano sino también a nivel de producción animal.

Al observar anualmente los casos ENO (2.800) y egresos hospitalarios (8.075), se visualiza una clara discrepancia. Según Pavletic ${ }^{19}$, corresponde a una subnotificación de casos ENO, lo cual difiere con lo planteado por Cortés ${ }^{20}$, la cual señala que existiría un sobrevaloración de los egresos hospitalarios, considerando que los casos ENO tienen diagnóstico confirmado:, sin embargo, llama la atención que sólo $11,7 \%$ son confirmados por serología y clínica; y 12,1\% por biopsia y clínica, por lo tanto sobre $80 \%$ de los casos ENO son confirmados sólo por aspecto clínico ${ }^{21}$. Adicionalmente a lo considerado en la publicación de Cortés, este estudio incorporó datos de mortalidad y AVPP, considerando un periodo más extenso y hasta donde se dispone de información en la actualidad.

En Chile, sólo se describe la especie E. granulosus ${ }^{22}$; no obstante, en el registro ENO se han confirmado 17 casos B67.4; 24 con B67.5; 9 como B67.6; y 7 con B67.7. Lo anterior da cuenta de la necesidad de la confirmación de caso por otros medios y no sólo clínica. Por lo tanto, se aprecia una falta de capacitación del equipo de salud involucrado en torno al tema del diagnóstico.

A partir de 2002 en egresos hospitalarios se registra la información sobre intervención quirúrgica. Si bien, la equinococosis quística ha sido considerada tradicionalmente de resolución quirúrgica, del período 2002-2008 (6.925), sólo 53,6\% presenta algún tipo de cirugía. Pavletic ${ }^{19}$ señala que aproximadamente $8 \%$ de los pacientes intervenidos por hidatidosis reingresan por una segunda intervención, por lo cual se mantendría una brecha importante entre los casos ENO y egresos hospitalarios.

Del total de egresos hospitalarios, 16,5\% (1.336) corresponden a < 15 años, lo que refleja infección reciente. Si bien, se ha producido una disminución proporcional desde 2001 (17,8\%) a 2008 (14,7\%), ésta no está del todo establecida dado que los datos 2008 son preliminares y en 2007 los < 15 años concentraron 18,9\%.

Si bien tanto los casos ENO como egresos hospitalarios presentan una tendencia a decrecer, se puede plantear la sub-notificación de casos ENO. En consecuencia, se desconoce el número real de casos anuales en el país, lo cual podría ser estimado mediante estudios de prevalencia serológica.

La región con mayor tasa de notificación ENO y egresos hospitalarios es Aysén- la segunda en mayor tasa de mortalidad- siendo por lo tanto, la región más afectada en el país. Coincide en ser la mayor zona de ganadería ovina a nivel país.

De acuerdo al tiempo de estadía, se observa una estabilidad en torno a 8-9 días, lo cual difiere significativamente de lo señalado por Pavletic ${ }^{20}$ para años anteriores, alrededor de 25-45 días, lo cual se explicaría por las mejoras en el tratamiento quirúrgico.

De acuerdo al análisis de la mortalidad, se aprecia que los afectados mayoritariamente son hombres adultos mayores con un nivel de escolaridad baja o ninguna, lo cual facilita la trasmisión de la enfermedad. 
Al observar la proporción de muertes en egresos hospitalarios se aprecia una tendencia al decrecimiento, lo cual concuerda con los avances en las técnicas quirúrgicas y su disponibilidad.

El país se ha visto impactado por esta zoonosis sobre la mortalidad; sin embargo, los AVPP han disminuido alrededor de 60,2\% entre 2001 (AVPP = 646,28) y 2008 (AVPP $=257,4)$. En general, los hombres perdieron más AVPP que las mujeres, lo cual es indicativo de la sobremortalidad masculina respecto de la mujer, lo que se manifiesta con un mayor número de muertes y AVPP a lo largo de la vida. Desde los 60 años, son las mujeres quienes pierden comparativamente más AVPP, esto último estaría explicado por la mayor esperanza de vida de la mujer, por lo cual sobrevive más años que el hombre.

En general, luego del análisis de estas diferentes fuentes de información, podemos señalar que el sistema de vigilancia epidemiológica debe ser sujeto a una evaluación en términos de su consistencia, exactitud, comparabilidad, validez, sensibilidad e integralidad. Además, debe ser reforzado con estudios de seroprevalencia que permitan cuantificar la magnitud de la enfermedad a nivel nacional.

Las defunciones por esta patología son evitables, por lo tanto es considerada una enfermedad postergada, donde se hace necesario abordar la situación en términos locales considerando las condiciones específicas de cada región, pero bajo un concepto de política pública nacional.
Agradecimientos: A Sra. Eugenia Guerra, Lucy Espinoza y María Isabel Silva. Departamento de Estadísticas e Información en Salud (DEIS). Ministerio de Salud de Chile.

\section{Resumen}

Objetivo: Caracterizar epidemiológicamente la hidatidosis humana en Chile. Material: Se utilizaron datos del Sistema de Notificación Obligatoria (2001-2009), Egresos hospitalarios (2001-2008), Defunciones (2001-2008) y Años de Vida Potencialmente Perdidos (2001-2008). Resultados: La incidencia promedio, según las notificaciones obligatorias fue 1,9 casos por 100 mil habs. (hombres 51,4\%). Edad mediana 38 años. Egresos hospitalarios presentaron una tasa promedio de 6,3 egresos por $100 \mathrm{mil}$ habs. El mayor diagnostico fue B67.9 (equinococosis, otra y la no especificada). La tasa de mortalidad promedio fue 0,2 defunciones por 100 mil habs. El nivel de instrucción de los afectados fue mayoritariamente básico. Se produjo la pérdida de 3.349 años de vida por el fallecimiento prematuro de 235 personas. Conclusiones: Las tasas de incidencia de casos notificados, egresos hospitalarios y mortalidad tienden a disminuir. Llama la atención la incongruencia entre los casos notificados y egresos hospitalarios, lo cual sugiere una sub-notificación. Se recomienda potenciar el sistema de notificación, confirmación de casos y estimular estudios de prevalencia.

\section{Referencias}

1.- Menezes da Silva A. Human Echinococcosis: a neglected disease. Gastroenterol Res Pract 2010;

2.- Organización Panamericana de la Salud (OPS). Organización Mundial de la Salud (OMS). Informe del Proyecto Subregional Cono Sur de Control y Vigilancia de la Hidatidosis Argentina, Brasil, Chile y Uruguay. Primera Reunión Constitutiva. Montevideo, Uruguay, 7 al 9 de julio de 2004.

3.- Amez J, Castañeda E. Tratamiento quirúrgico de la hidatidosis pulmonar en el Hospital Nacional Cayetano Heredia 1989-1999. Rev Med Hered 2002; 13 (1): 3-9.

4.- Osorio M, Godoy H. Estudio "Vulnerabilidad Social Frente a Hidatidosis Humana”. Informe Final Versión 2.0, Marzo 2008. Informe no publicado.

5.- Frider B, Larrieu E, Odrizola M. Long term outcome of a symptomatic liver hidatidosis. J Hepatol 1999; 30: 228-31.

6.- Manterola C, Vial M, Fonseca G, Carrasco R, Bustos L, Muñoz S, et al. Hidatidosis abdominal de localización extra hepática: características clínicas y evolución de una serie de casos. Rev Chil Cir 2002; 54 (2): 128-34.
7.- $\quad$ Franchi C, Divico B, Teggi A. Long-term evaluation of patients with hidatidosis treated with benzimidazole carbamatos. Clin Infect Dis 1999; 29. 304-9.

8.- Berns D, Rager B. Emerging infectious diseases: a cause for concern. Isr Med Assoc J 2000; 2: 919- 23.

9.- Guarnera E. Hidatidosis Humana, su Impacto, Diagnóstico, Manejo y Tratamiento. Organización Panamericana de la Salud (OPS). Organización Mundial de la Salud (OMS). Informe del Proyecto Subregional Cono Sur de Control y Vigilancia de la Hidatidosis Argentina, Brasil, Chile y Uruguay. Primera Reunión Constitutiva. Montevideo, Uruguay, 7 al 9 de julio de 2004, pp., 57-85.

10.- Irabedra P, Salvatella R. El Proyecto Subregional Cono Sur de Control y Vigilancia de la Hidatidosis. Rev Peru Med Exp Salud Pública. 2010; 27(4): 598-603.

11.- Campano S. Plan de acción y estrategias regionales básicas para la eliminación de la hidatidosis humana en América del Sur. Organización Panamericana de la Salud (OPS). Organización Mundial de la Salud (OMS). Informe del Proyecto Subregional Cono Sur de Control y Vigilancia de la Hidatidosis Argentina, Brasil, Chile y
Uruguay. Primera Reunión Constitutiva. Montevideo, Uruguay, 7 al 9 de julio de 2004, pp., 51-57.

12.- Gobierno de Chile. Ministerio de Salud. Reglamento sobre notificación de enfermedades transmisibles de declaración obligatoria $\mathrm{N}^{\circ} 158$. Santiago, 22 de octubre de 2004.

13.- Gobierno de Chile. Ministerio de Salud. Normas Técnicas de Vigilancia de Enfermedades Transmisibles. $2000<$ http://epi.minsal.cl/epi/ html/public/enftransmisibles.pdf > [accedido: 19 febrero de 2011].

14.- Rodríguez J. Hidatidosis humana: Enfermedad parasitaria se ha transformado en un serio problema de salud pública. El Rancagüino Online. 9 Noviembre de 2009 <http:// www.elrancaguino.cl/news/hidatidosishumanaenfermedad-parasitaria-se-hatransformado-en-un-serio-problema-de-saludpublica/> [accedido: 22 abril de 2010].

15.- Organización Panamericana de la Salud (OPS). Clasificación Estadística Internacional de Enfermedades y Problemas relacionados con la Salud. Décima revisión. 1995.

16.- Gobierno de Chile. Ministerio de Salud. Datos de Egresos Hospitalarios 2007.

17.- Gobierno de Chile. Ministerio de Salud. Esperanza de vida al nacer (en años) por período 
y sexo. Chile, 1950-2025<http://deis.minsal. cl/deis/ev/esperanza_de_vida/index.asp> [accedido: 20 febrero de 2011]

18.- Gobierno de Chile. Instituto Nacional de Estadística (INE). Departamento de Demografía. Programa de proyecciones de la población. Proyecciones de población por grupos de edad y sexo. <http://www.ine.cl/ canales/chile_estadistico/demografia_y_vitales/ proyecciones/MenPrincOK.xls> [accedido: 20 febrero de 2011].

19.- Pavletic C. Chile. Organización Panamericana de la Salud (OPS). Organización Mundial de la Salud (OMS). Informe del Proyecto Subregional Cono Sur de Control y Vigilancia de la Hidatidosis Argentina, Brasil, Chile y Uruguay. Primera Reunión Constitutiva. Montevideo, Uruguay, 7 al 9 de julio de 2004, pp., 34-38.

20.- Cortés S, Valle C. Hidatidosis humana:
Generalidades y situación epidemiológica en Chile según egresos hospitalarios y notificación obligatoria entre los años 2001 y 2005. Rev Chil Infectol 2010; 27 (4): 329-35.

21.- Gobierno de Chile. Ministerio de Salud. Base de datos Enfermedades de Notificación Obligatoria (ENO) 2001-2009.

22.- Muñoz P. Diagnóstico y tratamiento de la hidatidosis (comentario editorial). Rev Chil Infectol 2007; 24 (2): 153-4. 